\title{
Editorial
}

\section{Catalyzed Mizoroki-Heck Reaction or C-H Activation}

\author{
Sabine Berteina-Raboin (D) \\ Institut de Chimie Organique et Analytique (ICOA), Université d'Orléans UMR-CNRS 7311, BP 6759, \\ rue de Chartres, 45067 Orléans CEDEX 2, France; sabine.berteina-raboin@univ-orleans.fr
}

Received: 29 October 2019; Accepted: 30 October 2019; Published: 6 November 2019

In the last few decade, research conducted on the development by catalytic processes of C-C bonds formation on the one hand and on the other hand on the activation of $\mathrm{C}-\mathrm{H}$ bonds has grown considerably [1,2]. For their outstanding contribution to development of Palladium-Catalyzed cross-coupling reaction, Richard F. Heck with Akira Suzuki and Ei-ichi Negishi obtained the 2010 Nobel Prize in Chemistry. However, many improvements are still possible in terms of selectivity or even enantioselectivity via the development of new ligands or the study of the catalytic effect of other metals to carry out the same chemical transformations.

Zhang et al. emphasize, in their review [3], that the Mizoroki-Heck reaction is one of the most important catalytic methods to generate $\mathrm{C}-\mathrm{C}$ bonds in organic synthesis. This reaction is highly efficient and has good chemo- and stereoselectivity. The authors discuss the interest of using fluorine-containing agents as cross-coupling partners, in this reaction, to introduce fluorine atom(s) into organic molecules because these compounds show advantageous physicochemical and biological properties. However, only few organo-fluorinated natural compounds exist. The Mizoroki-Heck Cross-coupling reaction between fluorinated alkenes with aryl halide or equivalent and/or some alkenes with fluorinated aryl or alkyl halide or equivalent is the best pathway to obtain these fluorinated organic compounds in a view to drug discovery and advanced materials. This review presents the various combination of reagents usable with this process.

Sangeeta Jagtap [4] gives an overview of the state of the art of the Heck reaction. This reaction has played an important role in the elaboration of numerous compounds in many fields and is probably one of the most studied cross-coupling reactions in organic and medicinal chemistry. Sangeeta Jagtap has summarized many reviews on this topic mainly about catalysts, ligands and various conditions used but also suggested mechanisms.

Damien Prim's group worked on acridines, aza-polycyclic compound having a broad range of properties and applications in therapeutic, pigments, dyes, imaging probes, sensor and some other materials activities [5]. The 5,6-dihydrobenzo[c]acridine, comprises of four fused cycles of which one is partially hydrogenated leading to not fully planar compounds usable in the preparation of helical-shaped molecules. The authors succeeded to functionalize this kind of tetracyclic molecule by regioselective alkoxylation via C-H bond activation avoiding the standard pre-halogenation.

Berteina-Raboin's group developed the first direct $\mathrm{C}-\mathrm{H}$ arylation on $\mathrm{C} 2$ and $\mathrm{C} 3$ thiophene ring for a convenient one-pot synthesis of thienopyridine, thienopyrimidine, and thienopyrazine scaffolds [6].

The attention paid to environmentally friendly methods in terms of the quantities of catalysts, ligands and solvents is currently indispensable. In this context, Shi, Nawaz, Zaman and Sun [7] summarized recent advances in enantioselective C-H activation/functionalization via Mizoroki-Heck reaction or Suzuki reaction. These are methodologies that are used to generate synthetic, hemisynthetic or natural compounds with high added value whether in medicinal chemistry or agrochemistry. Conventional methods require pre-functionalization of the substrates which generates additional steps of synthesis and purification whereas the direct activation of the $\mathrm{C}-\mathrm{H}$ bond allows an atom-economy and a more sustainable chemistry. However, the direct activation of inert $\mathrm{C}-\mathrm{H}$ bonds still remains difficult because of the poor reactivity and selectivity. In their review the authors discuss the latest 
progress on enantioselective C-H activation via Mizoroki-Heck or Suzuki reaction with a particular interest on the origin of chirality and discussion on mechanisms with chiral ligands used.

Always in the interest of developing in a more environmentally sound manner, Berteina-Raboin's group [8] described the synthesis of the ABA phytohormone (abscisic acid) which exhibit some interesting biological activities and synthesis of new analogues performed solvent and ligand free Mizoroki-Heck reaction conditions. Some delicate dienes and trienes were obtained without isomerization in moderate to good yields (27-78\%). The phytohormone ABA was synthesized with this process in four steps from commercially available diketone in $54 \%$ global yields.

Finally, Mokhtar's team has developed a series of heterogeneous catalysts, the MgAl-layered double hydroxide, its calcined form at $500{ }^{\circ} \mathrm{C}$ (MgALOx) and the rehydrated form (MgAl-HT-RH) for the Henry reaction between nitroalkanes and various aldehydes [9]. This is the first study for understanding the effect of mesoporous and basic nature of this kind of catalysts for the Henri reaction. These catalysts have been fully characterized and the large surface area of mesoporous catalysts as well as the strong basic sites of rehydrated catalyst allowed a very efficient catalytic activity. In addition, the catalyst is reusable without loss of activity after five catalytic cycles what makes this process a green protocol.

This Special Issue on "Catalyze Mizoroki-Heck Reaction or C-H activation" was focussed on new advances in the formation of C-C bonds via the Mizoroki-heck reaction or new C-H activation methods. I would like sincerely thank all authors for their valuable contributions, original research papers and short reviews on synthesis of biologically active compounds using these catalytic processes, identification of new catalysts, of new conditions allowing selectivity or enantioselectivity, the activity and stability of catalysts under turnover conditions and all improvements in catalytic processes. I also sincerely thank the editorial team of Catalysts for their kind support and fast responses. Without you all, this special issue would not have been possible.

Excellent research is being performed worldwide on new processes to increase the efficiency of bioactive compounds elaboration and numerous efforts were made to develop sustainable chemistry but we only are at the beginning.

Conflicts of Interest: The author declares no conflicts of interest.

\section{References}

1. Kim, D.S.; Park, W.J.; Jun, C.H. Metal-Organic Cooperative Catalysis in C-H and C-C Bond Activation. Chem. Rev. 2017, 117, 8977-9015. [CrossRef] [PubMed]

2. Ritleng, V.; Sirlin, C.; Pfeffer, M. Ru-, Rh-, and Pd-Catalyzed C-C Bond Formation Involving C-H Activation and Addition on Unsaturated Substrates: Reactions and Mechanistic Aspects. Chem. Rev. 2002, 102, 1731-1770. [CrossRef] [PubMed]

3. Yang, J.; Zhao, H.W.; He, J.; Zhang, C.P. Pd-Catalyzed Mizoroki-Heck Reactions Using Fluorine-Containing Agents as the Cross-Coupling Partners. Catalysts 2018, 8, 23. [CrossRef]

4. Jagtap, S. Heck Reaction-State of the Art. Catalysts 2017, 7, 267. [CrossRef]

5. Campos, J.F.; Berteina-Raboin, S. The First Catalytic Direct C-H Arylation on C2 and C3 of Thiophene Ring Applied to Thieno-Pyridines, -Pyrimidines and -Pyrazines. Catalysts 2018, 8, 137. [CrossRef]

6. Large, B.; Bourdreux, F.; Damond, A.; Anne Gaucher, A.; Prim, D. Palladium-Catalyzed Regioselective Alkoxylation via C-H Bond Activation in the Dihydrobenzo[c]acridine Series. Catalysts 2018, 8, 139. [CrossRef]

7. Dumonteil, G.; Hiebel, M.-A.; Berteina-Raboin, S. Solvent-Free Mizoroki-Heck Reaction Applied to the Synthesis of Abscisic Acid and some Derivatives. Catalysts 2018, 8, 115. [CrossRef] 
8. Shi, S.; Nawaz, K.S.; Zaman, M.K.; Sun, Z. Advances in Enantioselective C-H Activation/Mizoroki-Heck Reaction and Suzuki Reaction. Catalysts 2018, 8, 90. [CrossRef]

9. Abdellattif, M.H.; Mokhtar, M. MgAl-Layered Double Hydroxide Solid Base Catalysts for Henry Reaction: A Green Protocol. Catalysts 2018, 8, 133. [CrossRef] 